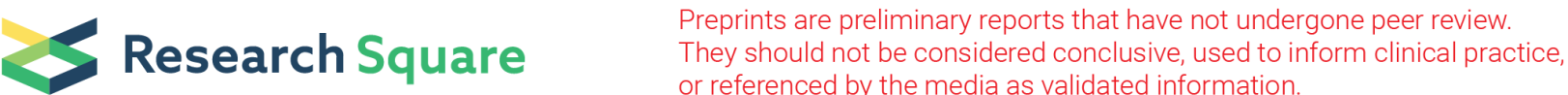

\section{Clinical and Epidemiological Characteristics and Outcome of Patients With Covid-19 in Sri Lanka; An Observational Study}

Meththananda Herath ( $\nabla$ herathtp@gmail.com )

University of Ruhuna Faculty of Medicine https://orcid.org/0000-0001-5853-8880

Jayasekera Madduma Mudiyanselage Priyamali thusharika

General Sir John Kotelawala Defence University

Pathirage Loku pathirage Manoji Muditha Kumari

University of Peradeniya Faculty of Medicine

Thushan Amila Wijesinghe Wedikkula Acharige

Base Hospital Kattankuddy

karthick Gunananthan

Base hospital Homagama

Bernard Pingamagae Don June

Iranawilla COVID treatment Center

Nirosha Madhuwanthi Hettiarachchi

Teaching Hospital Peradeniya

Thushari Dilrukshi Kaluthantrige Dona

Mulleriyawa Base Hospital

Piyarathne Lasantha Roshan Samarakoon Pathirana Mudiyanselage

Base Hospital Mullariyawa

Nuwan Bhathiya Senevirathne

Base Hospital Mulleriyawa

Sivajini Bhishman

Base Hospital Mulleriyawa

Asanka Gunaseela Jayawardana Kankanamage Aruna Jayawardhana Base Hospital Wallikanda

Harsha Priya Sanjeewa Weerasinghe arachchige

Base Hospital Wallikanda

Priyantha Balasooriya

navy general Hospital, Welisara

Prasath Thushanthy

Navy General Hospital Welisara

Ganaka Senarathna 
Karapitiya Teaching Hospital

Krishantha Jayasekera

Karapitiya Taching Hospital

\section{Sutharshan Ponnuthurai}

General Hospital Pollonnaruwa

\section{Suranga Ravinda Manilgama}

Teaching Hospital Kandy

Inoka Kumudini Jayasinghe

Teaching Hospital Kandy

Thilak Widana Arachchilage Ananda Jayalath

University of Peradeniya Faculty of Medicine

\section{Pirasath Selladurai}

Iranawilla COVID treatment center

\section{Research article}

Keywords: COVID-19, SARS-coV-2, HCQ use, asymptomatic rate

Posted Date: July 24th, 2020

DOI: https://doi.org/10.21203/rs.3.rs-42505/v1

License: (c) (1) This work is licensed under a Creative Commons Attribution 4.0 International License. Read Full License 


\section{Abstract \\ Background}

Clinical manifestations of coronavirus disease 2019 (COVID19) can vary from asymptomatic to profusely symptomatic due to many factors. Having data on the clinical characteristics of the affected patients is important for the screening and diagnosis of COVID-19. Main objective of this study is to evaluate the clinical and epidemiological characteristics and outcomes of patients with COVID-19 admitted to six treatment centres in Sri Lanka.

\section{Methods}

For the specific epidemic situation of COVID-19, we chose to conduct a descriptive retrospective study from six treatment centres in the country between March 10, 2020 and May 30, 2020.

\section{Results}

The study included 431 laboratory confirmed patients with COVID-19. Of them, 335 (78.2\%) were males and their mean age was 37 years. Most patients (373: 86.5\%) had had close contact with COVID-19 positive patients. The majority were asymptomatic (243: $56.3 \%)$ and of the symptomatic patients, the most common symptom was sore throat $(81,19 \%)$, followed by cough $(72,17 \%)$, headache $(72,17 \%)$, fever $(50,12 \%)$, and nasal discharge $(36,8 \%)$. Eight patients required either high dependency care or intensive unit care two of them succumbed to the illness ( $0.5 \%)$. Hydroxychloroquine (HCQ) was used as a treatment in 248 patients and they had a significantly longer (17.6 days) duration of hospital stays compared those who were not treated (13.6 days) with HCQ. When the HCQ was not used as a treatment, asymptomatic individuals had significantly shorter durations of hospital (11.1 days) stays compared to symptomatic individuals (14.3 days).

\section{Conclusions}

The majority of patients remained asymptomatic during the course of illness. The most common symptom was sore throat, seen in one fifth of patients, followed by cough, and headache. Our study also indicated the possibility of reduced viral clearance and hence longer duration of hospital stays with HCQ.

\section{Background}

The coronavirus disease has made a huge impact globally since late 2019. Pneumonia of unknown aetiology emerged from city of Wuhan, Hubei Province, China, in November 2019. Chinese scientists isolated the virus and it was named novel coronavirus 2019 (nCov-2019) (1). Later, the World Health 
Organization (WHO) reclassified this pathogen as severe acute respiratory syndrome coronavirus 2 (SARS-CoV-2) and the disease as Coronavirus Disease 2019 (COVID-19) (2).

As of June 30,2020, there are over 10 million confirmed cases and nearly 500,000 deaths reported worldwide (3). COVID-19 spreads by human-to-human transmission through droplets, faeco-oral routes, and direct physical contact (4). The incubation period of COVID-19 varies between 2 to 14 days(5). Most patients show clinical symptoms in 5 to 6 days(5). Noted epidemiological prognostic risk factors include being male, being older-aged (above 65 years), being a smoker, and having obesity, diabetes, chronic pulmonary diseases, hypertension, cardiovascular diseases, and chronic kidney disease. The disease is more severe when more risk factors are observed at presentation(6). A wide array of clinical features is shown in patients with COVID-19. Clinical manifestations can be classified as asymptomatic or symptomatic, carrier or infective state, and ranging from mild prodrome to profusely symptomatic(5). The usual triad of symptoms at the time of presentation are fever, dry cough, and dyspnoea(5). Several factors affect the clinical picture including the virulence of the strain of the virus and host factors such as age, immunity status and patient comorbidities(7). The Chinese Center for Disease Control and Prevention has described the epidemiological characteristics of patients in their case series consisting of 72,314 COVID-19 patients. The majority exhibited a mild level of disease (81\%) while $14 \%$ had moderate to severe disease conditions and $5 \%$ were critically ill(8).

On January 27, 2020, the first case was reported in Sri Lanka. It was an imported case: a Chinese tourist. The first confirmed local case was reported on March 11,2020. Since then, the number of cases has increased gradually. As of June 30,2020, the number of confirmed cases is 2,033, out of which 1,661 have completely recovered. Only 11 deaths have been reported(9). In Sri Lanka, diagnosed patients are managed according to the local guidelines in designated treatment hospitals for COVID-19 islandwide(10).

\section{Rationale}

Only limited publications on COVID-19 in local contexts are available. The interim analysis on clinical and epidemiological characteristics of the confirmed first 100 COVID-19 patients from Sri Lanka following their discharge/death is one of the important literatures among them(11). The lack of availability of data highlighted the importance and necessity of researching this valuable information. As a result, the Sri Lanka College of Internal Medicine designed this multi-centre study in designated treating centres of COVID-19 patients to cover the whole island.

Clinical and epidemiological characteristics, morbidity, and mortality of COVID-19 patients can vary from country to country. Various studies from many parts of the globe have reported different prominent clinical characteristics $(5,12-15)$. People belonging to certain ethnic groups might be at an increased risk of acquisition, disease severity, and poor outcomes in COVID-19 for a variety of reasons(16). The factors that may influence clinical features and outcomes are ethnicity, mean age of population, geographical region, humidity, climate, and other country-specific factors such as overcrowding, and most importantly the circulating strain of the virus $(17,18)$. For example, South Asians usually have higher rates of non- 
communicable diseases (NCDs) such as diabetes mellitus, hypertension, and cardiovascular diseases, which are associated with severe disease and mortality in COVID-19(16). Furthermore, ethnicity could interact with the spread of the virus through factors such as lower socioeconomic statuses, culture, religion, and behavioural factors(16). Therefore, there is an urgent need to study the epidemiological and clinical characteristics of patients with COVID-19 in Sri Lanka. It is also important to study outcomes such as mortality, the number of patients requiring oxygen therapy and intensive care unit (ICU) care, and the total durations of hospital stays which may be unique to Sri Lankan patients with COVID-19.

The objectives of this study is to evaluate the clinical and epidemiological characteristics and outcomes of patients with COVID-19 admitted to six treatment centres in Sri Lanka.

\section{Methods}

Study design

For the specific epidemic situation of COVID-19, we chose to conduct a descriptive retrospective study from six treatment centres in the country, namely Base Hospital Walikanda, Base Hospital Mulleriyawa, COIVD Treatment Centre Iranawilla, Base Hospital Kattankudy, Base Hospital Homagama, and Navy General Hospital, Welisara.

Data was extracted from patients' notes to collect socio-demographic factors, clinical characteristics of the presenting illness, laboratory investigations, management including treatment with Hydroxychloroquine (HCQ), and the outcome of the illness.

\section{Eligibility criteria}

Medical notes and compiled data of any patients with laboratory-confirmed COVID-19 admitted to any of the above six treatment centres between March 10, 2020 and May 30, 2020 were included in the study. All patients (symptomatic as well asymptomatic) admitted these six treatment centres were kept under close medical observation till they become asymptomatic and two consecutive PCR become negative. A confirmed patient of COVID-19 is defined as a positive result on high-throughput sequencing or real-time reverse-transcriptase-polymerase-chain-reaction (RT-PCR) assay of nasal and pharyngeal swab specimens. The National Institute for Infectious Disease (NIID) Hospital, Colombo was excluded from the analysis due to practical reasons of difficulty in accessing patients' records.

\section{Data collection}

Data extraction from medical records and accuracy was confirmed by a consultant physician or acting consultant physician attached to each of the six treatment centres. A Google Form was used to collect the data which was pretested with the patients recorded data of 25. Ethical clearance and administrative clearance were obtained from the Ethical Review Committee General Sir John Kotelawala Defence University, and the Education, Training and Research Unit, Ministry of Health Sri Lanka, respectively. 


\section{Statistical Analysis}

Continuous variables with a normal distribution were expressed as mean (SD) with a non-normal distribution as median with IQR and categorical variables were expressed as counts and percentages. Descriptive analysis was carried out as the chosen cohort of patients was a convenient selection. However, comparisons of values between HCQ treated and non-treated groups were performed using a Student's $t$ test after confirming the normal distribution. The data was analysed using the Statistical Package for the Social Sciences (SPSS) software version 24.0.

\section{Results}

A total of 431 PCR positive COVID-19 subjects were studied. Of them, 335 (78\%) were males and 96 $(22 \%)$ were females. The mean age was 37.1 years, with the mean age for males being 35 years and the mean age of females being 45 years. Of this cohort, a majority (292) were Sinhalese, followed by Muslims (116), and Tamils (23). Half of the patients (219:50.81\%) were navy personnel who were employed by the Sri Lanka Navy while only two police personnel and one army personnel were present.

Table 1

baseline characteristics based on presence or absence of symptoms

\begin{tabular}{|llll|}
\hline & Symptomatic $\mathbf{n = 1 8 8}$ & Asymptomatic $\mathbf{n}=\mathbf{2 4 3}$ & All \\
\hline Mean age (years, SD) & $36.7(12.1)$ & $37.8(13.6)$ & $37.1(13.2)$ \\
\hline Gender & $\mathrm{N}(\%)$ & $\mathrm{N}(\%)$ & $335(77)$ \\
\hline Male & $174(86.1)$ & $161(70.9)$ & $96(23)$ \\
\hline Female & $29(13.8)$ & $67(29.1)$ & \\
\hline Co-morbidities (all = 89) & $\mathrm{N}(\%)$ & $\mathrm{N}(\%)$ & $31(7.1)$ \\
\hline Diabetes mellitus & $13(6.4)$ & $18(7.9)$ & $43(9.9)$ \\
\hline Hypertension & $18(8.9)$ & $25(11)$ & $14(3.2)$ \\
\hline Asthma & $8(3.9)$ & $6(2.6)$ & $5(1.2)$ \\
\hline Ischemic heart disease & $0(0)$ & $5(2.2)$ & $13(3)$ \\
\hline Dyslipidaemia & $5(2.5)$ & $8(3.5)$ & \\
\hline
\end{tabular}

Most patients (373: 86.5\%) had close contact with COVID-19 positive patients. Of them, $52.4 \%$ (226) patients declared their close contact as occurring in their workplaces. Fourteen patients (3.2\%) had been exposed to the overseas returnees and only three patients claimed that they may have contracted the virus at the airport. There were 35 patients $(8.1 \%)$ with a positive history of overseas travel within the previous three months before they got infected. Seventy-three (16.9\%) patients were identified during contact tracing and interestingly, there was no obvious known contact history in 58 patients $(13.4 \%)$. 
A majority 342 (79.4\%) had no significant medical comorbidities and of the 89 patients with comorbidities. The most common comorbidity was $(43,9.9 \%)$ was hypertension, followed by diabetes (31, 7.1\%), and dyslipidaemia (14, 3.2\%) (Table 1). There were 271 smokers.

Table 2

Symptoms on admission and symptoms developed after admission

\begin{tabular}{|c|c|c|c|}
\hline \multirow[t]{3}{*}{ Symptom } & $\begin{array}{l}\text { Symptoms appeared before } \\
\text { admission }\end{array}$ & $\begin{array}{l}\text { Symptoms appeared after } \\
\text { admission }\end{array}$ & \multirow[t]{2}{*}{$\begin{array}{l}\text { All } \\
N=431\end{array}$} \\
\hline & $N=87$ & $N=101$ & \\
\hline & n (\%) & n (\%) & n (\%) \\
\hline Fever & $27(6.2)$ & $23(5.3)$ & $\begin{array}{l}50 \\
(11.6)\end{array}$ \\
\hline Cough & $39(9.0)$ & $33(7.6)$ & $\begin{array}{l}72 \\
(16.7)\end{array}$ \\
\hline Headache & 37 (8.6) & $35(8.1)$ & $\begin{array}{l}72 \\
(16.7)\end{array}$ \\
\hline Arthralgia & $10(2.3)$ & $13(3.0)$ & $23(5.3)$ \\
\hline Myalgia & $23(5.3)$ & $16(3.7)$ & $39(9.0)$ \\
\hline Sore throat & $40(9.3)$ & $41(9.5)$ & $\begin{array}{l}81 \\
(18.8)\end{array}$ \\
\hline Nasal discharge & $15(3.5)$ & $21(4.9)$ & $36(8.6)$ \\
\hline Fatigability & $3(0.7)$ & $10(2.3)$ & $13(3.0)$ \\
\hline Diarrhoea & $14(3.2)$ & $7(1.6)$ & $21(4.9)$ \\
\hline $\begin{array}{l}\text { Shortness of } \\
\text { breath }\end{array}$ & $9(2.1)$ & $3(0.6)$ & $12(2.8)$ \\
\hline Anosmia & $6(1.4)$ & $4(0.9)$ & $10(2.3)$ \\
\hline
\end{tabular}

The presenting symptoms and the symptoms developed while under medical observation are shown in Table 2. On admission, the majority were asymptomatic (344:80\%) with only $20 \%$ having symptoms either before or on admission. During the period of medical observation in the hospital, another 101 patients $(23.4 \%)$ developed symptoms. Out of the symptomatic patients, the most common presenting symptom was sore throat $(81,19 \%)$, followed by cough $(72,17 \%)$, headache $(72,17 \%)$, fever $(50,12 \%)$ and nasal discharge $(36,8 \%)$. Only $10(2 \%)$ had anosmia. Seven patients $(1.6 \%)$ had dyspnoea on admission with another 15 (3.5\%) developed dyspnoea during the hospital stay. Only five patients developed hypoxia during their hospital stay that needed oxygen therapy. The mean pulse rate was 82 beats/minute and the average blood pressure was $124 / 74 \mathrm{mmHg}$. 
The mean white cell count (WCC) of our study group was $7.9 \times 10^{9 /} \mathrm{L}$ with a mean neutrophil count of 4.4 $\times 10^{9} / \mathrm{L}$ and a lymphocyte count of $2.83 \times 10^{9} / \mathrm{L}$. Only a $49(11 \%)$ had thrombocytopenia. The mean platelet count was $264 \times 10^{9} / \mathrm{L}$ and the mean C-reactive protein (CRP) was $5.88 \mathrm{mg} / \mathrm{L}$.

Table 3

-Blood cells versus symptomatic and asymptomatic illness

\begin{tabular}{|llll|}
\hline & $\begin{array}{l}\text { Symptomatic } \mathbf{n}= \\
\mathbf{1 8 8}\end{array}$ & $\begin{array}{l}\text { Asymptomatic } \mathbf{n}= \\
\mathbf{2 4 3}\end{array}$ & All \\
\hline Blood cells & $\mathrm{N}(\mathrm{sd})$ & $\mathrm{N}(\mathrm{sd})$ & $\mathrm{N}(\mathrm{sd})$ \\
\hline $\begin{array}{l}\text { Total White-cell count } \\
\left(\text { cells } / \mathrm{mm}^{3}\right)\end{array}$ & $7843(2898)$ & $8100(2014)$ & $7945(2453)$ \\
\hline Neutrophil count $\left(\mathrm{cells} / \mathrm{mm}^{3}\right)$ & $4278(2452)$ & $4552(1620)$ & $4451(1930)$ \\
\hline Lymphocyte count $\left(\mathrm{cells} / \mathrm{mm}^{3}\right)$ & $2949(1487)$ & $2795(845)$ & $2834(1202)$ \\
\hline Platelet count/ $\mathrm{\mu L}$ & $254514(84532)$ & $279119(80137)$ & 264562 \\
\hline
\end{tabular}

Out of 431 patients, only three patients required high dependency care and five were transferred to the intensive care unit. Two patients died due to pneumonia complicated with acute respiratory distress syndrome (ARDS). Eight patients were transferred to the National Institute of Infectious Disease (NIID), Colombo.

Table - 4 Mean duration of hospital stay (days) versus treatment with HCQ

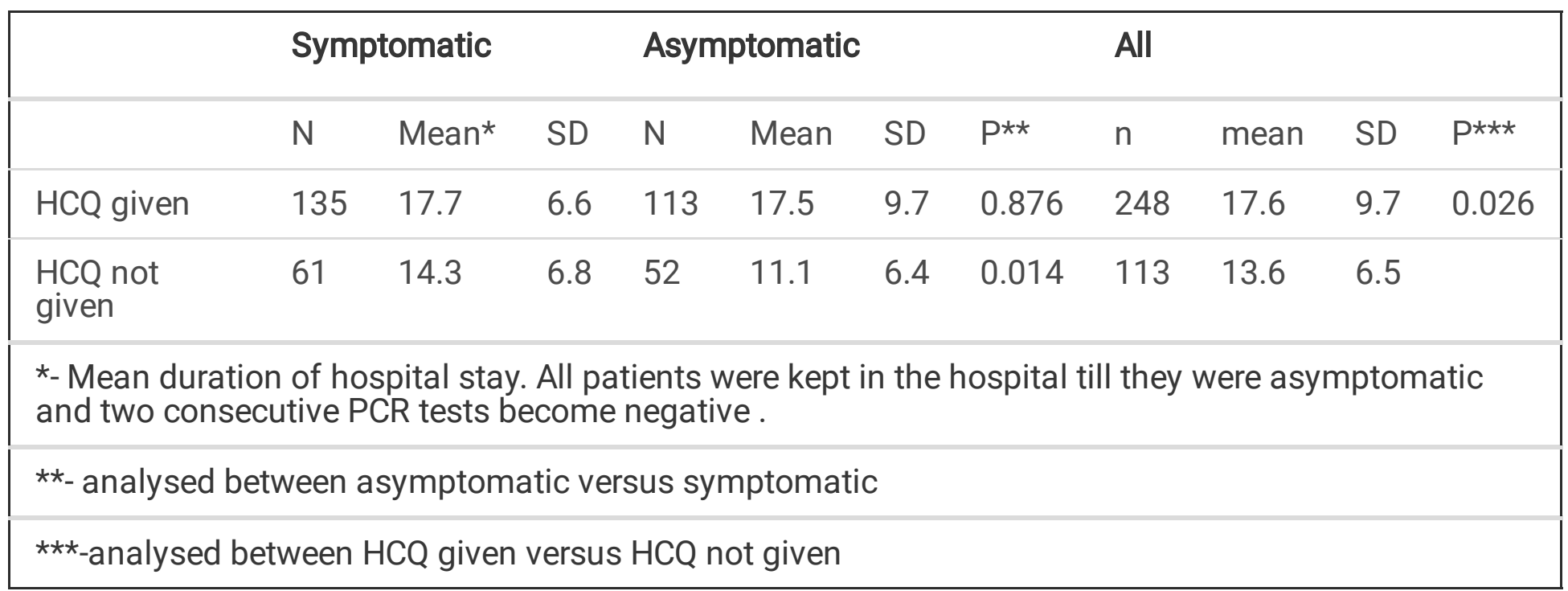

Information on HCQ used as a treatment was available in 361 patients (Table 4). Of them, those who were treated with HCQ $(248,69 \%)$ had a significantly longer (17.6 days) durations of hospital stays compared those who were not treated (13.6 days) with HCQ. Furthermore, both asymptomatic and symptomatic patients treated with HCQ had similar and longer durations of hospital stays than the 
patients not treated with HCQ. When the HCQ was not used as a treatment, asymptomatic individuals had a significantly shorter duration of hospital (11.1 days) stay compared to symptomatic individuals (14.3). This might suggest that HCQ had negative effect on viral clearance among both asymptomatic and symptomatic individuals with COVID-19.

\section{Discussion}

Sri Lanka is considered to be one of the few countries in the world which has successfully controlled the spread of COVID-19. Sri Lanka, from the outset implemented a strict lockdown involving the closure of schools and non-essential workplaces, a ban on social gatherings, and severe travel restrictions by imposing an island-wide curfew. These strict measures from the beginning of the outbreak enabled the country to limit the spread of the disease with a comparatively low disease burden with a very low mortality rate. As a result of these strict measures, the pattern and the clinical characteristics of COVID-19 in Sri Lanka may have taken a different shape and therefore are worth reporting(15). In addition, through these measures Sri Lanka mitigated COVID-19 in a unique way, where all the positive patients with COVID-19 were admitted to dedicated COVID-19 hospitals and kept under strict observation till their recovery and confirmation of "virus-negative" status. This unique situation allowed us to retrospectively observe the clinical course of all positive patients, asymptomatic as well as symptomatic with the SARSCoV-2 infection.

Most of our patients were recognised when they were asymptomatic during the process of contact tracing and screening. All positive patients were hospitalized and the majority (54\%) of them remained asymptomatic during the course of the illness. The higher proportion of asymptomatic patients in our study contrasts with the findings from other studies. Guan et al. reported fever in $88.7 \%$ of patients in 1,099 hospitalised patients in China at the outset of outbreak of COVID-19(5). Among 104 people with the SARS-CoV-2 infection on the Diamond Princess cruise ship, a majority (68\%) had symptoms(19). Similarly, a study conducted in a tertiary care hospital in Pune, India had a higher proportion (75\%) of symptomatic cases(20). The most common symptom in our study was sore throat (19\%) followed by cough (17\%), and headache (17\%). These findings contrast with the findings of previously published studies of COVID-19. In most study series, fever was the most common symptom, followed by cough(5, $13,19)$. Interestingly, most published studies reported sore throat as a less common symptom, reported only in $13.5 \%$ by Guan et al in China, $23.8 \%$ by Nitesh Gupta et al in India, whereas in our study sore throat is the most common symptom $(5,21)$. Another notable finding was the absence of fever in the majority of our patients (88\%) (376/431). Similar to other published studies, runny nose is a comparatively rare symptom (8\%) in this study(5).

There are a number of possible reasons for the contrasting pattern of symptomatology in this study compared to the most published studies from other part of the world. One explanation could be patients' characteristics such as age. In our study the mean age is 37 years with nearly $50 \%$ being otherwise healthy and physically fit navy personnel. These young and healthy individuals are more likely to develop asymptomatic infection with less prominent symptoms than older individuals $(5,19)$. Intensive contact 
tracing and screening helped in recognising more patients with asymptomatic illnesses in our study. Other possibilities include a less virulent viral strain causing COVID-19 in Sri Lanka, the effect of geographical factors such as higher temperatures, and prevention of repeated re-exposure to the virus due to intensive screening and isolation.

The demographic factors causing symptomatic illness among individuals infected with SARS-CoV-2 and risk factors for disease progression in COVID-19 are still not clear as health systems in most countries actively discourage individuals with no or mild symptoms from admitting themselves into a hospital. However, as all individuals found positive for the SARS-CoV-2 infection were admitted to a hospital in Sri Lanka (regardless of symptoms), we were able to retrospectively observe the clinical course of asymptomatic people with the SARS-CoV-2 infection. In this study $20 \%(87 / 431)$ had symptoms at the time of admission and another 23\% (101/431) subsequently developed symptoms (pre-symptomatic illness). More than half $(56 \%, 243 / 431)$ remained asymptomatic during the extended period of close medical observation, and never exhibited any symptoms. Of the patients who became symptomatic during their hospital stay, the most common symptoms were sore throat $(9.5 \%)$, and headache $(8 \%)$ followed by cough (7.6\%) and fever (5.3\%). These findings contrast with the findings of China where the prevalence of "true" asymptomatic infections was found to be in the range of 1.5 to $2.8 \%(5,8)$. However, this figure is clearly an underestimate since testing has primarily occurred only among individuals who have symptoms.

The study which most closely resembles this study is the study conducted on people infected on board the Diamond Princess cruise ship. In this study, the true prevalence of asymptomatic infection was $32 \%$ (22). However, the median age of people in this study was 64 years with half having comorbidities whereas in this study the mean age was 37 years. The younger subjects and significantly lower number of comorbidities would be the likely reasons for the higher prevalence of asymptomatic infections in the present study(23). A study conducted by Meyers KJ et al on asymptomatic individuals with COVID-19 revealed $71 \%$ remained asymptomatic during the follow up period(24). Similarly, our study also showed a true asymptomatic rate of $71 \%$ (244/345) among 345 asymptomatic individuals on admission. Based on the results of our study and the study conducted by Meyers et al we can conclude that around $70 \%$ of asymptomatic individuals with SARS-CoV-2 infection don't develop any symptoms.

Other interesting finding observed in our study was the negative the effect of HCQ on the duration of SARS-CoV-2 positivity and hence duration of hospital stays. Our study revealed that those who were treated with HCQ had had significantly longer (17.6 days) durations of hospital stays compared those who were not treated (13.6 days) with HCQ. Furthermore, both asymptomatic and symptomatic patients treated with HCQ had similar and longer durations of hospital stays than the patients not treated with HCQ. When the HCQ was not used as a treatment, asymptomatic individual had significantly shorter duration of hospital (11.1 days) stay compared to symptomatic individuals (14.3 days). This finding possibly indicates that HCQ might lead to reduced viral clearance preferentially among asymptomatic individuals, prolonging duration of SARS-CoV-2 positivity. A study conducted by Mallat et al showed that patients not treated with HCQ had viral clearance within a shorter duration (10 days) compared to HCQ 
treated patients (17 days)(25). To our knowledge there were no previous study comparing the effect of HCQ among symptomatic versus asymptomatic individuals with COVID-19.

The major limitation of this study was the inability to include patients with COVID-19 admitted to one of the major COVID-19 dedicated treatment hospital NIID at Colombo due to practical reasons. We enrolled

all patients referred to all the other six dedicated COVID-19 treatment hospitals (available at the time of study) in Sri Lanka. As patient referrals to these hospitals was not randomly based it is possible that patients with severe symptoms would have been admitted preferentially to the NIID. Having a large proportion of otherwise healthy navy personnel in our study certainly have affected the final results. Therefore, the patient population was not an exact representation of normal public settings. Additionally, some laboratory tests as well as images (chest radiograph) were obtained only for those with symptoms and blood tests were done only once for most patients, and thus we were unable to determine changes in lymphocyte count and radiological findings over the course of observation.

\section{Conclusions}

Our study showed that Sri Lankan patients with COVID-19 have different clinical characteristic than reported previously. Sore throat was the most common symptom; however, more than half of patients were completely asymptomatic. Our study further showed that a majority of asymptomatic patients at the time of admission remained asymptomatic (true asymptomatic) causing no significant morbidity or mortality. Furthermore, the large proportion of asymptomatic cases indicates the difficulty in identifying patients with COVID-19 without intensive contact tracing and screening. Therefore, all countries that successfully controlled the spread of SARS-CoV-2 infection have to be extremely vigilant about a resurgence of infection that could be sparked by undetected transmission by asymptomatic and presymptomatic individuals. This finding emphasizes the importance of intensive contact tracing and screening to control the spread of SARS-CoV-2 infection in the community. Our study also indicated the possibility of reduced viral clearance with HCQ in vivo among asymptomatic individuals with COVID-19.

\section{Declarations}

Ethics approval and consent to participate

This study is approved by the Ethical Review Committee of Faculty of Medicine, Kothalawala Defence University, Werahera, Sri Lanka .

Consent for publication

Not applicable as this is a retrospective study

Competing interests

All the authors declare that they have no competing interests. 
Funding

This study was not funded and authors of the study covered the expenses .

Authors' contributions

HMMH, JWATA, GS, KJ and JMMPT conceived and designed the study. HNM, MSR, JIK and HMMH contributed to the literature search. GK, PDJB , KDTD, SB, WAHPS, BP, JGJKAA, PS, TP, WWATA, SNB, SPMLRP, SPMLRP and SP contributed to the clinical patient care, management and data collection. $\mathrm{HMMH}, \mathrm{JMMPT}$, PLPMMKP, GS, and HNM contributed to the manuscript preparation. HMMH, PLPMMKP contributed to the data analysis. JWATA contributed to the manuscript verification. All authors have read and approved the manuscript.

Availability of data and materials

The datasets used and/or analysed during the current study are available from the corresponding author on reasonable request.

Acknowledgements

The authors are very grateful to the Ministry of Health, Sri Lanka and all the directors in the six treatment centres for their kind corporation throughout the study. We wish to acknowledge Dr MA Sachinthya Nethmini Wickramasinghe and Dr RW RumaduChampani for their assistance in preparing the manuscript.

Conflict of interest : All authors declare that they have no conflict of interest.

\section{References}

1. Chen Y, Liu Q, Guo D. Emerging coronaviruses: Genome structure, replication, and pathogenesis. 2020;92(4):418-23.

2. Singhal T. A Review of Coronavirus Disease-2019 (COVID-19). Indian J Pediatr. 2020;87(4):281-6.

3. Coronavirus. update(live) wordometer, https://www.worldometers.info/ /cornonavirus.

4. Wang Y, Wang Y, Chen Y, Qin Q. Unique epidemiological and clinical features of the emerging 2019 novel coronavirus pneumonia (COVID-19) implicate special control measures. 2020;92(6):568-76.

5. Guan WJ, Ni ZY, Hu Y, Liang WH, Ou CQ, He JX, et al. Clinical Characteristics of Coronavirus Disease 2019 in China. N Engl J Med. 2020;382(18):1708-20.

6. Singh A, Shaikh A, Singh R, Singh AK. COVID-19: From bench to bed side. Diabetes metabolic syndrome. 2020;14(4):277-81.

7. Chen N, Zhou M, Dong X, Qu J, Gong F, Han Y, et al. Epidemiological and clinical characteristics of 99 cases of 2019 novel coronavirus pneumonia in Wuhan, China: a descriptive study. Lancet. 2020;395(10223):507-13. 
8. Wu Z, McGoogan JM. Characteristics of and Important Lessons From the Coronavirus Disease 2019 (COVID-19) Outbreak in China: Summary of a Report of 72314 Cases From the Chinese Center for Disease Control and Prevention. Jama. 2020.

9. Epidemiology, Unit. Coronavirus 2020, Situation Reports. Colombo: Ministry of Health, 2020 http://wwwepidgovlk/web/indexphp?lang=en Accessed on 20 May 2020.

10. Ceylon College of Physicians and Epidemiology Unit. Provisional Clinical Guidelines on COVID-19 suspected and confirmed patients. Colombo: Ministry of Health (Accessed on 20 May 20). https://ccp.lk/wp-content/uploads/2013/04/COVID-19-CPG-VERSION-5-30.04.2020_Draft-min.pdf.

11. Clinical and Epidemiological Characteristics of the Confirmed COVID-19. Patients in Sri Lanka. Status Report Based on the Interim Analysis of the First 100 COVID-19 Patients Following Hospital Discharge or Death. Ministry of Health and Indigenous Medical Services, Colombo, Sri Lanka, 10th May 2020.

12. Young BE, Ong SWX, Kalimuddin S, Low JG, Tan SY, Loh J, et al. Epidemiologic Features and Clinical Course of Patients Infected With SARS-CoV-2 in Singapore. Jama. 2020;323(15):1488-94.

13. Lechien JR, Chiesa-Estomba CM, Place S, Van Laethem Y, Cabaraux P, Mat Q, et al. Clinical and epidemiological characteristics of 1420 European patients with mild-to-moderate coronavirus disease 2019. Journal of internal medicine. 2020.

14. Aggarwal S, Garcia-Telles N, Aggarwal G, Lavie C, Lippi G, Henry BM. Clinical features, laboratory characteristics, and outcomes of patients hospitalized with coronavirus disease 2019 (COVID-19): Early report from the United States. Diagnosis (Berlin Germany). 2020;7(2):91-6.

15. Bielecki M, Züst R, Siegrist D, Meyerhofer D, Crameri GAG, Stanga ZG, et al. Social distancing alters the clinical course of COVID-19 in young adults: A comparative cohort study. Clinical infectious diseases: an official publication of the Infectious Diseases Society of America; 2020.

16. Pareek M, Bangash MN, Pareek N, Pan D, Sze S, Minhas JS, et al. Ethnicity and COVID-19: an urgent public health research priority. Lancet. 2020;395(10234):1421-2.

17. Sajadi MM, Habibzadeh P, Vintzileos A, Shokouhi S, Miralles-Wilhelm F, Amoroso A. Temperature, Humidity, and Latitude Analysis to Estimate Potential Spread and Seasonality of Coronavirus Disease 2019 (COVID-19). JAMA Netw Open. 2020;3(6):e2011834-e.

18. O'Reilly KM, Auzenbergs M, Jafari Y, Liu Y, Flasche S, Lowe R. Effective transmission across the globe: the role of climate in COVID-19 mitigation strategies. Lancet Planet Health. 2020;4(5):e172-e.

19. Tabata S, Imai K, Kawano S, Ikeda M, Kodama T, Miyoshi K, et al. Clinical characteristics of COVID-19 in 104 people with SARS-CoV-2 infection on the Diamond Princess cruise ship: a retrospective analysis. Lancet Infect Dis. 2020:S1473-3099(20)30482-5.

20. Tambe MP, Parande MA, Tapare VS, Borle PS, Lakde RN, Shelke SC. An epidemiological study of laboratory confirmed COVID-19 cases admitted in a tertiary care hospital of Pune, Maharashtra. Indian J Public Health. 2020;64(Supplement):183-s7.

21. Gupta N, Agrawal S, Ish P, Mishra S, Gaind R, Usha G, et al. Clinical and epidemiologic profile of the initial COVID-19 patients at a tertiary care centre in India. Monaldi archives for chest disease = 
Archivio Monaldi per le malattie del torace. 2020;90(1).

22. Mizumoto K, Kagaya K, Zarebski A, Chowell G. Estimating the asymptomatic proportion of coronavirus disease 2019 (COVID-19) cases on board the Diamond Princess cruise ship, Yokohama, Japan, 2020. Euro Surveill. 2020;25(10):2000180.

23. Liu Y, Mao B, Liang S, Yang J-W, Lu H-W, Chai Y-H, et al. Association between age and clinical characteristics and outcomes of COVID-19. Eur Respir J. 2020;55(5):2001112.

24. Meyers KJ, Jones ME, Goetz IA, Botros FT, Knorr J, Manner DH, et al. A cross-sectional communitybased observational study of asymptomatic SARS-CoV-2 prevalence in the greater Indianapolis area. Journal of medical virology. 2020.

25. Mallat J, Hamed F, Balkis M, Mohamed MA, Mooty M, Malik A, et al. Hydroxychloroquine is associated with slower viral clearance in clinical COVID-19 patients with mild to moderate disease: A retrospective study. medRxiv. 2020:2020.04.27.20082180. 